\title{
Force-detected nuclear double resonance between statistical spin polarizations
}

\author{
M. Poggio ${ }^{1,2}$, H. J. Mamin ${ }^{1}$, C. L. Degen ${ }^{1}$, M. H. Sherwood ${ }^{1}$, and D. Rugar ${ }^{1}$ \\ ${ }^{1}$ IBM Research Division, Almaden Research Center, 650 Harry Rd., San Jose CA \\ ${ }^{2}$ Center for Probing the Nanoscale, \\ Stanford University, 476 Lomita Hall, Stanford CA
}

(Dated: April 18, 2022)

\begin{abstract}
We demonstrate nuclear double resonance for nanometer-scale volumes of spins where random fluctuations rather than Boltzmann polarization dominate. When the Hartmann-Hahn condition is met in a cross-polarization experiment, flip-flops occur between two species of spins and their fluctuations become coupled. We use magnetic resonance force microscopy to measure this effect between ${ }^{1} \mathrm{H}$ and ${ }^{13} \mathrm{C}$ spins in ${ }^{13} \mathrm{C}$-enriched stearic acid. The development of a cross-polarization technique for statistical ensembles adds an important tool for generating chemical contrast in nanometer-scale magnetic resonance.

PACS numbers: 76.70.-r, 05.40.-a, 76.60.-k, 76.60.Pc
\end{abstract}


The physics of microscopic spin ensembles can be distinctly different from that of macroscopic ensembles. For example, in volumes of nuclear spins smaller than about $(100 \mathrm{~nm})^{3}$, random spin flips generate a fluctuating polarization that exceeds the typical thermal (or Boltzmann) polarization [1, 2, 3]. These spin fluctuations are a major source of dephasing in solid-state quantum systems [4, 5], and their control is an important prerequisite for nanometer-scale magnetic resonance imaging (MRI) and spectroscopy [6, 7, 8, 9, 10, 11]. Recent experiments using magnetic resonance force microscopy (MRFM) [12, 13] have extracted useful information from random polarization and harnessed it for nanometer-scale threedimensional imaging [7]. One way to further improve nanometer-scale MRI is to combine its imaging capability with the chemical selectivity intrinsic to magnetic resonance. Techniques such as nuclear magnetic resonance (NMR) spectroscopy routinely exploit this feature for the structural analysis of molecules in millimeter-sized samples. At the nanometer-scale, the same information could be used to locally probe the chemical composition of materials, and to identify specific molecules in complex biological structures.

Here we apply nuclear double resonance to achieve such a form of contrast using crosspolarization $(\mathrm{CP})$ between statistically polarized ${ }^{1} \mathrm{H}$ and ${ }^{13} \mathrm{C}$ spins in ${ }^{13} \mathrm{C}$-enriched stearic acid. CP is widely used in NMR for the signal enhancement of low-abundance and low- $\gamma$ nuclei and forms the basis for many advanced multidimensional spectroscopy techniques [14]. Indeed, CP has been demonstrated as an efficient chemical contrast mechanism for micrometer-scale one-dimensional MRFM imaging based on Boltzmann polarization [15, 16, 17]. This spectroscopic method is not directly applicable to statistically polarized volumes of spins since at any given time the polarization has a random sign and magnitude, making measured signals intrinsically irreproducible. One way around this problem, as demonstrated here, is to observe the change in the correlation time of the fluctuations $[3,18]$.

CP relies on matching the rotating-frame Zeeman splittings of two different spin species (denoted as $I$ and $S$ ) in order to promote cross-species spin flip-flops through the heteronuclear dipolar coupling [14]. For this purpose, two strong rf fields are applied with frequencies near the Larmor resonance of the respective spins. According to the original work by Hartmann and Hahn, efficient transfer is achieved when the rf field strengths are such that the respective Rabi frequencies have a similar magnitude [14, 19]. The Rabi frequency is determined by the effective field in the rotating frame; for a spin $I$, the effective field is given by $\mathbf{B}_{\text {eff, } I}=B_{1 I} \hat{x}^{\prime}+\frac{2 \pi}{\gamma_{I}} \Delta \nu_{I} \hat{z}$, where $B_{1 I}$ is the magnitude and $\Delta \nu_{I}=\nu_{I}-\frac{\gamma_{I}}{2 \pi} B_{0}$ is the reso- 
nance offset of the rf field with a frequency $\nu_{I} \cdot \gamma_{I}$ is the nuclear gyromagnetic ratio of $I$, $\hat{x}^{\prime}$ is a unit vector in the rotating frame, and $\hat{z}$ is a unit vector along the static field $\mathbf{B}_{0}$ (likewise for the $S$ spin). The Hartmann-Hahn (HH) condition can then be expressed as $\gamma_{I}\left|\mathbf{B}_{\text {eff,II }}\right|=\gamma_{S}\left|\mathbf{B}_{\text {eff,S }}\right|$. The efficiency of CP depends on the angles of the effective fields with respect to the $\hat{x}^{\prime}$ axis in the respective rotating frames, so that the most efficient transfer occurs when $\Delta \nu_{I}=\Delta \nu_{S}=0$ and $\gamma_{H} B_{1 H}=\gamma_{C} B_{1 C}$ [15]. For samples with a high spin density and strong nuclear moments, the transfer process is typically very efficient, occurring on a characteristic time-scale set by the dipolar coupling frequency between spins. In stearic acid it is estimated at about $16 \mu \mathrm{s}[20,21]$.

For Boltzmann polarizations, a spin temperature description is commonly used in which the two spin polarizations are viewed as thermal ensembles [22]. This model successfully explains the dynamics of CP at the macroscopic level as well as in micrometer-scale MRFM [16]. When the $\mathrm{HH}$ condition is met, the two spin ensembles come into thermal contact and their temperatures equilibrate. In this way, one thermal ensemble can be used to enhance or deplete the polarization of the other ensemble.

For statistically polarized nuclear spins, the same exchange of polarization occurs, except that the mean polarization of both spin ensembles is zero. When there is no HH contact, the polarization of an ensemble in the rotating frame fluctuates about zero with a variance that is given by the number of spins in that ensemble. The fluctuations in each ensemble occur on a time-scale determined by that ensemble's rotating frame relaxation rate. When the $\mathrm{HH}$ condition is met, double resonance allows rapid flip-flop processes that exchange polarization between the $I$ and $S$ spin ensembles, while simultaneously conserving the total polarization. As a result, fluctuations can occur on a much faster time-scale.

To obtain a more quantitative picture, we consider the case for a spin- $1 / 2$ system where the polarizations of the ensembles are represented by $n_{I}$ and $n_{S}$, defined as the difference between spin-up and spin-down populations for the $I$ and $S$ spins respectively. The dynamics of the HH transfer can then be described by a set of detailed balance equations that use spin population difference instead of spin temperature (see Ref. [19], Eq. (8)),

$$
\begin{aligned}
& \frac{\partial n_{I}}{\partial t}=-k_{I} n_{I}-\frac{k_{I S}}{N}\left(N_{S} n_{I}-N_{I} n_{S}\right)+r_{I}(t)+r_{I S}(t), \\
& \frac{\partial n_{S}}{\partial t}=-k_{S} n_{S}+\frac{k_{I S}}{N}\left(N_{S} n_{I}-N_{I} n_{S}\right)+r_{S}(t)-r_{I S}(t),
\end{aligned}
$$

where $N$ is the total number of spins in the ensemble, $N_{I}$ and $N_{S}$ are the number of $I$ and 
$S$ spins respectively $\left(N=N_{I}+N_{S}\right), k_{I}$ and $k_{S}$ are the spin-lattice relaxation rates, and $k_{I S}$ is the average rate of exchange between $I$ and $S$ spins.

The original work by Hartmann and Hahn (Ref. [19], Eq. (8)) does not account for statistical spin fluctuations. To describe the random excitations that lead to statistical polarization, we therefore introduce three stochastic functions $r_{I}(t), r_{S}(t)$ and $r_{I S}(t)$, equivalent to the number of $I$-spin flips, $S$-spin flips, and cross-species spin flip-flops per unit time, respectively. We assume white spectral densities for these functions and further require that on sufficiently long time-scales, the variance of the fluctuating polarizations is equal to the number of spins in the ensemble [1, 11]. The resulting (double-sided) spectral densities are $S_{r_{I}}=2 k_{I} N_{I}$ and $S_{r_{S}}=2 k_{S} N_{S}$. The spectral density $S_{r_{I S}}=2 k_{I S} N_{I} N_{S} / N$ is similarly obtained [23].

The coupled differential equations (1) and (2) can be solved in frequency space, yielding expressions for the spectral densities of $n_{I}$ and $n_{S}$. One can then calculate experimental parameters, such as the variance or the characteristic time-scale of the spin fluctuations in the presence of $\mathrm{CP}$. We concentrate our analysis on the spectral density of the $I$-spin fluctuations:

$$
S_{n_{I}}=\frac{2 N_{I}\left[\left(k_{I}+k_{I S} \frac{N_{S}}{N}\right) \omega^{2}+\left(k_{S}+k_{I S} \frac{N_{I}}{N}\right)\left[k_{I} k_{S}+k_{I S}\left(k_{I} \frac{N_{I}}{N}+k_{S} \frac{N_{S}}{N}\right)\right]\right]}{\omega^{4}+\left[k_{I}^{2}+k_{S}^{2}+k_{I S}^{2}+2 k_{I S}\left(k_{I} \frac{N_{S}}{N}+k_{S} \frac{N_{I}}{N}\right)\right] \omega^{2}+\left[k_{I} k_{S}+k_{I S}\left(k_{I} \frac{N_{I}}{N}+k_{S} \frac{N_{S}}{N}\right)\right]^{2}} .
$$

Evaluation of (3) allows us to determine the behavior of the $I$-spin fluctuations for arbitrary values of $k_{I}, k_{S}$ and $k_{I S}$. We consider two particularly relevant cases. As expected, in the regime of negligible $\mathrm{CP}$ where $k_{I S} \ll k_{I}, k_{S}$, the $I$-spin fluctuations occur on a time-scale $\tau_{I}=k_{I}^{-1}$ with a variance equal to $N_{I}: \lim _{\substack{k_{I S} / k_{I} \rightarrow 0 \\ k_{I S} / k_{S} \rightarrow 0}} S_{n_{I}}=\frac{2 \tau_{I} N_{I}}{1+\omega^{2} \tau_{I}^{2}}$.

In the regime of strong $\mathrm{CP}$ where $k_{I S} \gg k_{I}, k_{S}$, two time-scales emerge. Rapid polarization transfer between spin ensembles leads to fast spin fluctuations with a characteristic time $\tau_{I S}=k_{I S}^{-1}$ and a variance $N_{I} N_{S} / N$. The reduction of the variance compared to $N_{I}$ occurs because the total polarization is conserved on time-scales short compared to $\tau_{I}$ and $\tau_{S}=k_{S}^{-1}$, thereby limiting the phase space of possible states. On top of the rapid exchange of polarization between spin ensembles, the polarization also fluctuates on a much slower time-scale $\tau_{\text {avg }}=\left(k_{I} \frac{N_{I}}{N}+k_{S} \frac{N_{S}}{N}\right)^{-1}$ with a variance given by $N_{I}^{2} / N$. Note that the sum of the rapid and slow variances is once again $N_{I}$. It is not surprising, then, that in this strong $\mathrm{CP}$ limit the $I$-spin spectral density approaches the sum of two spectral densities, each with 
one of the two characteristic times and variances:

$$
\lim _{\substack{k_{I} / k_{I S} \rightarrow 0 \\ k_{S} / k_{I S} \rightarrow 0}}\left[S_{n_{I}}\right]=\lim _{\substack{k_{I} / k_{I S} \rightarrow 0 \\ k_{S} / k_{I S} \rightarrow 0}}\left[\frac{2 \tau_{I S} N_{I} N_{S} / N}{1+\omega^{2} \tau_{I S}^{2}}+\frac{2 \tau_{a v g} N_{I}^{2} / N}{1+\omega^{2} \tau_{a v g}^{2}}\right] .
$$

Thus, the main signature of CP is the presence of spin fluctuations faster than $\tau_{I}$. Simple coin-flipping simulations support these findings.

We demonstrate nuclear $\mathrm{CP}$ between statistically polarized ${ }^{1} \mathrm{H}$ and ${ }^{13} \mathrm{C}$ spins in an experiment using a custom-built magnetic resonance force microscope [18]. For these species, where $\gamma_{H} / \gamma_{C}=3.9772$, the combination of the minimum $B_{1 I}$ needed for adiabatic inversions and the maximum allowed current in our microfabricated rf field source prevent us from reaching the condition of most efficient $\mathrm{CP}, \gamma_{H} B_{1 H}=\gamma_{C} B_{1 C}$. Nevertheless, we are able to observe significant $\mathrm{CP}$ at non-zero resonance offsets where the $\mathrm{HH}$ condition is met.

The sample is a $10-\mu$ m-sized particle of stearic acid, $\mathrm{C}_{18} \mathrm{H}_{36} \mathrm{O}_{2}$, where $>99 \%$ of the carbon is ${ }^{13} \mathrm{C}$. The particle is placed upon an ultrasensitive, single-crystal Si cantilever (120 $\mu \mathrm{m}$ long, $3 \mu \mathrm{m}$ wide, and $0.1 \mu \mathrm{m}$ thick), with part of the particle sticking out beyond the end of the cantilever. The cantilever and particle are then briefly heated to a few ${ }^{\circ} \mathrm{C}$ below the melting point promoting adhesion to the cantilever. The cantilever with the sample attached is then mounted in a vacuum chamber (pressure $<1 \times 10^{-6}$ torr) at the bottom of a cryostat, which is isolated from environmental vibrations. At the operating temperature of $4.2 \mathrm{~K}$, the sample-loaded cantilever has a resonant frequency $f_{0}=1 / T_{0}=2.9 \mathrm{kHz}$, an intrinsic quality factor $Q_{0}=44000$, and a spring constant $k=86 \mu \mathrm{N} / \mathrm{m}$. We actively damp the cantilever in order to give it a fast response time of $\sim 25 \mathrm{~ms}$. An FeCo nanomagnetic tip is used to produce the large $\left(\sim 10^{6} \mathrm{~T} / \mathrm{m}\right)$ spatial field gradient required for generating magnetic forces of a few attonewtons between the spins in the sample and the tip. A microwire underneath the tip generates an rf field of a few millitesla that induces magnetic resonance in the sample [18]. The MRFM measurement is carried out on a stearic acid sample positioned $100 \mathrm{~nm}$ above the magnetic tip in an externally applied magnetic field $\left|\mathbf{B}_{\text {ext }}\right|=2.64 \mathrm{~T}$.

We measure the spin polarization by periodically inverting the nuclei of choice using adiabatic rapid passages $[11,18]$. We operate in a fixed magnetic field $\mathbf{B}_{0}=\mathbf{B}_{\text {ext }}+\mathbf{B}_{t i p}$ $\left(\left|\mathbf{B}_{0}\right|=2.72 \mathrm{~T}\right)$, where $\mathbf{B}_{\text {tip }}$ is the field produced by the magnetic tip. We periodically sweep the frequency $\nu_{I}$ of a transverse rf magnetic field $\mathbf{B}_{1 I}$ through the Larmor resonance condition, $\nu_{I}=\frac{\gamma_{I}}{2 \pi} B_{0}$ so as to induce adiabatic inversions of the nuclear spin polarization. In the presence of the magnetic tip, periodic inversions of the spin polarization generate an 
alternating force that drives the mechanical resonance of the cantilever. The amplitude of cantilever oscillation, which we measure using optical interferometry, is then proportional to the $I$-spin polarization. As shown in Fig. 1, we can measure either the ${ }^{1} \mathrm{H}$ or the ${ }^{13} \mathrm{C}$ statistical polarization by adjusting the rf center frequency of the adiabatic passages.

In order to observe polarization transfer, we perform adiabatic passages on one isotope the "observed" or $I$-spin isotope. This measurement produces a signal that is proportional to the fluctuating $I$-spin polarization. Simultaneously, we address the other isotope - the "unobserved" or $S$-spin isotope — with cw radiation at or near its Larmor frequency. This continuously applied resonant $B_{1 S}$ is constant in the rotating frame and remains locked to a statistical polarization of $S$ spins with a correlation time $\tau_{S}$. The basic scheme is shown in Fig. 2.

When the $\mathrm{HH}$ condition is fulfilled, polarization transfer occurs between the statistically polarized "observed" and "unobserved" spin ensembles. Measurements are shown in Fig. 3 for both ${ }^{1} \mathrm{H}$ and ${ }^{13} \mathrm{C}$ in the role of the "observed" isotope. In both experiments we increment the resonance offset $\Delta \nu_{S}$ of the $S$ spins while detecting the $I$ spins with frequency sweeps centered on $\Delta \nu_{I}=0$. We record both the signal variance and the correlation time of the $I$-spin fluctuations. Dips in the correlation time appear at those frequencies where $\Delta \nu_{S}$ satisfies the $\mathrm{HH}$ condition. Since the signal is recorded in a narrow band $(\sim 17 \mathrm{~Hz})$ around the cantilever resonance, a reduction in the correlation time due to $\mathrm{CP}$ also gives rise to a reduction in the observed force signal.

Fig. 3 shows two distinct CP regimes. In the first regime, shown in Fig. 3(a), we observe the ${ }^{1} \mathrm{H}$ spins, using $\gamma_{H} B_{1 H}=280 \mathrm{kHz}$ and $\gamma_{C} B_{1 C}=29 \mathrm{kHz}$, so that $\gamma_{I} B_{1 I}>\gamma_{S} B_{1 S}$. Again, as we increment $\Delta \nu_{C}, \gamma_{C}\left|\mathbf{B}_{\text {eff,C }}\right|$ comes to a minimum at resonance according to $\gamma_{C}\left|\mathbf{B}_{\text {eff,C }}\right|=\sqrt{\left(\gamma_{C} B_{1 C}\right)^{2}+\left(2 \pi \Delta \nu_{C}\right)^{2}}$. For two bands of $\Delta \nu_{C}$ symmetric about zero, $\gamma_{C}\left|\mathbf{B}_{\text {eff,C }}\right|$ intersects the trajectory of $\gamma_{H}\left|\mathbf{B}_{\text {eff,H }}\right|$ thereby fulfilling the HH condition [16]. The resulting $\mathrm{CP}$ produces the double-dip structure shown in Fig. 3(a). The most efficient CP and therefore the most significant reduction in the ${ }^{1} \mathrm{H}$ correlation time (i.e. the deepest part of the dips) occurs for a $\mathrm{HH}$ match at the vertex of the $\gamma_{H}\left|\mathbf{B}_{\text {eff,H }}\right|$ hyperbola shown in Fig. 3(a). At these intersections the slopes of $\gamma_{C}\left|\mathbf{B}_{\text {eff,C }}\right|$ and $\gamma_{H}\left|\mathbf{B}_{\text {eff,H }}\right|$ match, producing the longest possible $\mathrm{HH}$ contact. In addition, $\mathrm{CP}$ is most efficient when $\Delta \nu_{C}$ and $\Delta \nu_{H}$ are smallest resulting in small angles of the effective field.

In the second regime, shown in Fig. 3(b), we observe the ${ }^{13} \mathrm{C}$ spins using $\gamma_{H} B_{1 H}=120$ 
$\mathrm{kHz}$ and $\gamma_{C} B_{1 C}=62 \mathrm{kHz}$, so that $\gamma_{I} B_{1 I}<\gamma_{S} B_{1 S}$. As we increment $\Delta \nu_{H}, \gamma_{H}\left|\mathbf{B}_{\mathrm{eff}, \mathrm{H}}\right|$ comes to a minimum at resonance according to $\gamma_{H}\left|\mathbf{B}_{\text {eff, } \mathrm{H}}\right|=\sqrt{\left(\gamma_{H} B_{1 H}\right)^{2}+\left(2 \pi \Delta \nu_{H}\right)^{2}}$. In this case, there is one band of $\Delta \nu_{H}$ symmetric about zero for which $\gamma_{H}\left|\mathbf{B}_{\text {eff,H }}\right|$ intersects the trajectory of $\gamma_{C}\left|\mathbf{B}_{\text {eff,C }}\right|$. The resulting CP produces the single-dip structure of Fig. 3(b). For the same reasons which apply in the first regime, the most efficient $\mathrm{CP}$ occurs for $\Delta \nu_{H}=0$ where the $\mathrm{HH}$ match is closest to the vertex of the $\gamma_{C}\left|\mathbf{B}_{\text {eff,C }}\right|$ hyperbola. The same single- and doubledip behavior shown in Fig. 3 appears in Fig. 2 of the original paper on CP by Hartmann and Hahn [19].

We have measured a series of spectra of the double-dip type for different $\gamma_{H} B_{1 H}$ and $\gamma_{C} B_{1 C}$. Typical results are shown in Fig. 4(a). Using simple Lorentzian fits to determine the position of the dips, we extract the splitting for all spectra. From the condition $\gamma_{I}\left|\mathbf{B}_{\mathrm{eff}, \mathrm{I}}\right|=$ $\gamma_{S}\left|\mathbf{B}_{\text {eff,S }}\right|$ and as pointed out by Hartmann and Hahn (Ref. [19], Eq. (75)), the dips in Fig. 4(a) should appear at

$$
\Delta \nu_{C}= \pm \Delta \nu_{C, H H} \equiv \pm \sqrt{\frac{\gamma_{H}^{2}}{4 \pi^{2}} B_{1 H}^{2}-\frac{\gamma_{C}^{2}}{4 \pi^{2}} B_{1 C}^{2}},
$$

so that the splitting is $2 \Delta \nu_{C, H H}$. The experimental data in Fig. $4(\mathrm{~b})$ and (c) agree within the error with the theoretical curve representing (5) without any adjustable parameters. Note that the magnitudes of $B_{1 H}$ and $B_{1 C}$ are calibrated by independent nutation experiments on each species [18]. Given this agreement, we confirm that our double-resonance features result from the $\mathrm{HH}$ effect.

While in our experiments it was possible to observe the ${ }^{13} \mathrm{C}$ signal directly, doubleresonance detection is particularly useful when one isotope with spin $S$ has a weak resonance signal, either because of a small $\gamma_{S}$ or a low abundance. The presence of the $S$ spins can then be detected via the stronger signal of the $I$ spins. The ability to perform $\mathrm{CP}$ in statistical ensembles provides the possibility of new contrast mechanisms for nanoscale MRI applications. For example, organic material with many proximate ${ }^{13} \mathrm{C}$ and ${ }^{1} \mathrm{H}$ atoms could be distinguished from interstitial water molecules, which possess only ${ }^{1} \mathrm{H}$. Statistical double resonance could be combined with advanced spectroscopy techniques for chemical characterization of materials at the nanometer-scale. Ultimately, such techniques could be applied to discern individual protein components in complex nanometer-scale biological structures.

We acknowledge support from the NSF-funded Center for Probing the Nanoscale (CPN) 
at Stanford University.

[1] F. Bloch, Phys. Rev. 70, 460 (1946).

[2] T. Sleator, E. L. Hahn, C. Hilbert, and J. Clarke, Phys. Rev. Lett. 55, 1742 (1985).

[3] H. J. Mamin, R. Budakian, B. W. Chui, and D. Rugar, Phys. Rev. B 72, 024413 (2005).

[4] L. Childress et al., Science 314, 281 (2006).

[5] D. J. Reilly et al., Science 321, 817 (2008).

[6] H. J. Mamin, M. Poggio, C. L. Degen, and D. Rugar, Nat. Nano. 2, 301 (2007).

[7] C. L. Degen et al., Proc. Nat. Acad. Sci. U.S.A., in press.

[8] C. L. Degen, Appl. Phys. Lett. 92, 243111 (2008).

[9] J. R. Maze et al., Nature 455, 644 (2008).

[10] G. Balasubramanian et al., Nature 455, 648 (2008).

[11] C. L. Degen, M. Poggio, H. J. Mamin, and D. Rugar, Phys. Rev. Lett. 99, 250601 (2007).

[12] D. Rugar, R. Budakian, H. J. Mamin, and B. W. Chui, Nature 430, 329 (2004).

[13] S. Kuehn, S. A. Hickman, and J. A. Marohn, J. Chem. Phys. 128, 052208 (2008).

[14] C. P. Slichter, Principles of Magnetic Resonance, 3rd ed. (Springer, New York, 1990), p.247ff.

[15] Q. Lin et al., Phys. Rev. Lett. 96, 137604 (2006).

[16] K. W. Eberhardt et al., Phys. Rev. B 75, 184430 (2007).

[17] K. W. Eberhardt, C. L. Degen, A. Hunkeler, and B. H. Meier, Angew. Chem. Int. Ed. 47, 8961 (2008).

[18] M. Poggio et al., Appl. Phys. Lett. 90, 263111 (2007).

[19] S. R. Hartmann and E. L. Hahn, Phys. Rev. 128, 2042 (1962).

[20] This estimate results from the rms average of $\mathrm{C}-\mathrm{H}$ dipolar half-splittings in a $\mathrm{CH}_{2}$ group taken over a powder distribution of field orientations. We use a $\mathrm{C}-\mathrm{H}$ bond length of $1.1 \AA$ and a $\mathrm{H}-\mathrm{C}-\mathrm{H}$ angle of $109.5^{\circ}$ for the calculation.

[21] W. Xiaoling, Z. Shanmin, and W. Xuewen, Phys. Rev. B 37, 9827 (1988).

[22] C. P. Slichter, Principles of Magnetic Resonance, 3rd ed. (Springer, New York, 1990), p.219ff.

[23] When the total polarization $n_{I}+n_{S}=0$, the probability distribution for a given value of $n_{I}$ is given by the product of two binomial distributions, which can be approximated by Gaussians with variances $N_{I}$ and $N_{S}$ respectively. The resulting distribution is another Gaussian with 
variance $N_{I} N_{S} /\left(N_{I}+N_{S}\right)$. 
Figures 


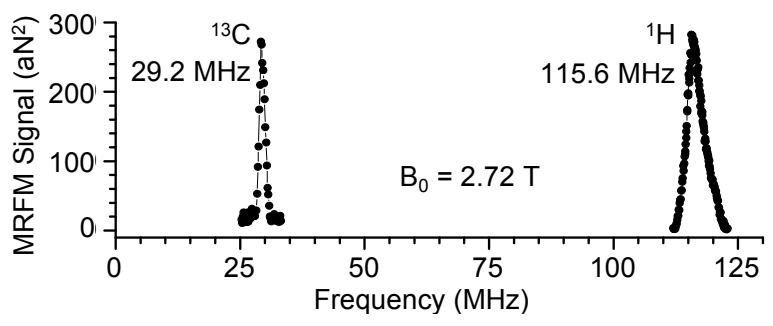

FIG. 1: MRFM signal as a function of rf frequency at $B_{0}=2.72 \mathrm{~T}$ for both the ${ }^{1} \mathrm{H}$ and ${ }^{13} \mathrm{C}$ isotopes in $99 \%{ }^{13} \mathrm{C}$-enriched stearic acid. The two data sets were taken under slightly different experimental conditions, making their relative amplitudes arbitrary. 


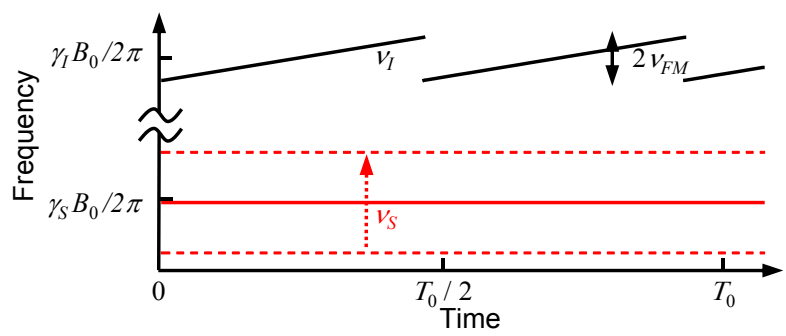

FIG. 2: A schematic diagram showing the rf frequencies $\nu_{I}$ and $\nu_{S}$ during an experiment. With $\nu_{S}$ constant, periodic sweeps of $\nu_{I}$ through resonance adiabatically invert the $I$ spins. The effect of incrementing $\nu_{S}$ is represented by the dotted arrow. 

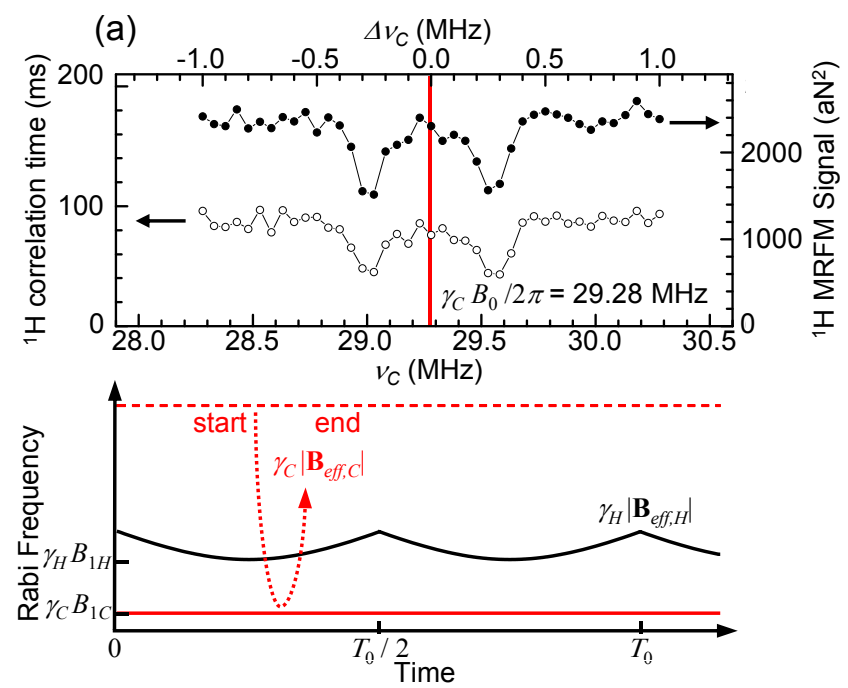

(b) $\quad \Delta v_{H}(\mathrm{MHz})$
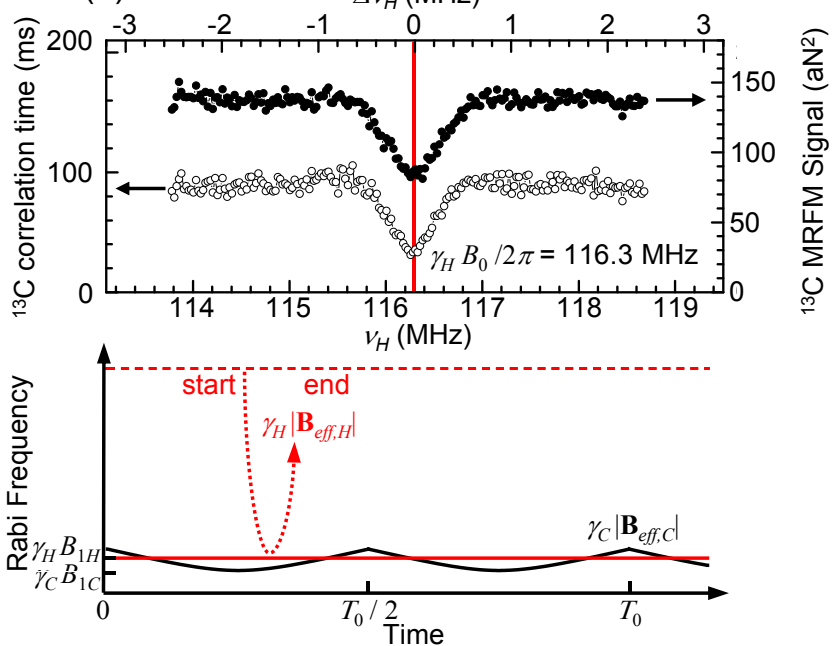

FIG. 3: CP resonances detected by MRFM in statistical polarizations plotted as a function of the $S$-spin resonance offset. Below each plot, the corresponding trajectories of the effective field Rabi frequencies are shown. The effect of scanning $\Delta \nu_{S}$ is to vertically shift $\gamma_{S}\left|\mathbf{B}_{\text {eff,S }}\right|$ as shown by the dotted arrow. (a) The "observed" spin $I={ }^{1} \mathrm{H}$ and the "unobserved" spin $S={ }^{13} \mathrm{C}$ with $\nu_{F M}=300$ kHz. (b) The "observed" spin $I={ }^{13} \mathrm{C}$ and the "unobserved" spin $S={ }^{1} \mathrm{H}$ with $\nu_{F M}=125 \mathrm{kHz}$. 

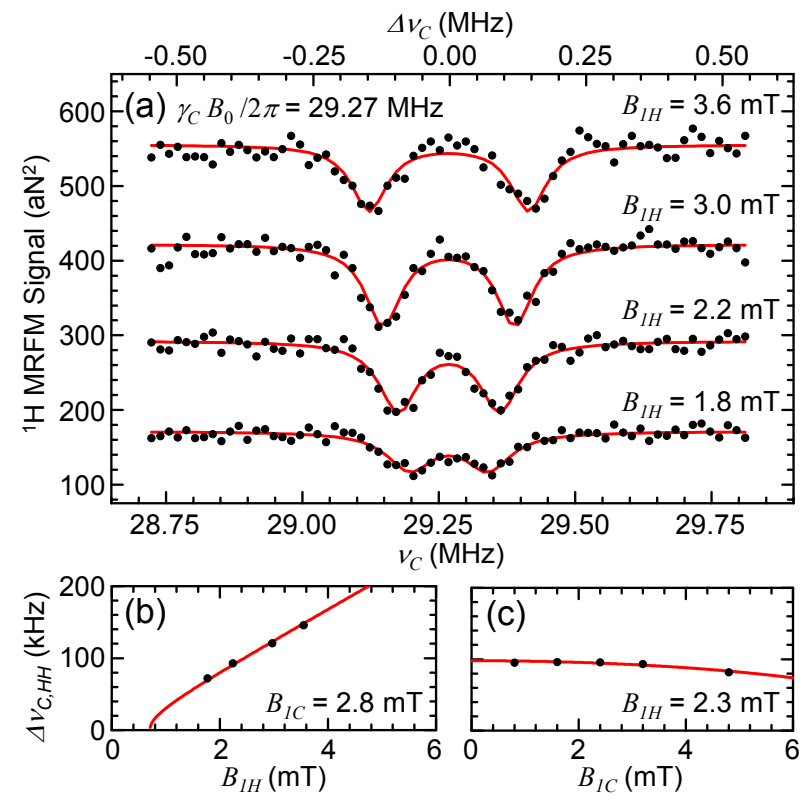

FIG. 4: (a) MRFM data (points) and fits (lines) as a function of the $S$-spin resonance offset for $I={ }^{1} \mathrm{H}$ and $S={ }^{13} \mathrm{C}$ for different $B_{1 H}$, with $B_{1 C}=2.8 \mathrm{mT}$. (b) $\Delta \nu_{C, H H}$ plotted as points for different $B_{1 H}$ taken from fits in (a). (c) $\Delta \nu_{C, H H}$ for different $B_{1 C}$ from fits to a separate data set (not shown). Lines in (b) and (c) correspond to (5) with no free parameters. 